\title{
Erratum to: Graphene coherent states
}

Erik Díaz-Bautista ${ }^{\mathrm{a}}$ and David J. Fernández ${ }^{\mathrm{b}}$

Physics Department, Cinvestav, P.O. Box. 14-740, 07000 Mexico City, Mexico

Original article: Eur. Phys. J. Plus (2017) 132: 499, https://doi.org/10.1140/epjp/i2017-11794-y

Received: 8 February 2019

Published online: 1 April 2019

(C) Società Italiana di Fisica / Springer-Verlag GmbH Germany, part of Springer Nature, 2019

After publication, the authors realized that eqs. (28a)-(28d), along with ref. [47] needed to be corrected. The following is the correct version.

Gamma functions in the denominators of the sums should appear as radicands in square roots. So, they should read as follows:

$$
\begin{aligned}
\langle\hat{z}\rangle_{\alpha} & =\frac{\sqrt{2} \mathbf{R e}(\alpha)}{2 \exp \left(r^{2}\right)-1}\left[\exp \left(r^{2}\right)+\sum_{n=1}^{\infty} \frac{r^{2 n}}{\sqrt{\Gamma(n) \Gamma(n+2)}}\right], \\
\left\langle\hat{z}^{2}\right\rangle_{\alpha} & =\frac{1}{4 \exp \left(r^{2}\right)-2}\left[1+4 r^{2} \exp \left(r^{2}\right)+2\left[[\operatorname{Re}(\alpha)]^{2}-[\mathbf{I m}(\alpha)]^{2}\right]\left(\exp \left(r^{2}\right)+\sum_{n=1}^{\infty} \frac{\sqrt{n+1} r^{2 n}}{\sqrt{\Gamma(n) \Gamma(n+3)}}\right)\right], \\
\langle\hat{p}\rangle_{\alpha} & =\frac{\sqrt{2} \mathbf{I m}(\alpha)}{2 \exp \left(r^{2}\right)-1}\left[\exp \left(r^{2}\right)+\sum_{n=1}^{\infty} \frac{r^{2 n}}{\sqrt{\Gamma(n) \Gamma(n+2)}}\right], \\
\left\langle\hat{p}^{2}\right\rangle_{\alpha} & =\frac{1}{4 \exp \left(r^{2}\right)-2}\left[1+4 r^{2} \exp \left(r^{2}\right)-2\left[[\operatorname{Re}(\alpha)]^{2}-[\operatorname{Im}(\alpha)]^{2}\right]\left(\exp \left(r^{2}\right)+\sum_{n=1}^{\infty} \frac{\sqrt{n+1} r^{2 n}}{\sqrt{\Gamma(n) \Gamma(n+3)}}\right)\right] .
\end{aligned}
$$

Since the plots associated with eqs. (28a)-(28d) were drawn with the correct expressions, the results in the original paper are still correct.

Moreover, ref. [47] was incorrect. Its correct version is presented here.

\section{References}

47. V. Jakubsky, M. Tusek, Ann. Phys. 378, 171 (2017).

Publisher's Note The EPJ Publishers remain neutral with regard to jurisdictional claims in published maps and institutional affiliations.

\footnotetext{
a e-mail: ediaz@fis.cinvestav.mx

b e-mail: david@fis.cinvestav.mx
} 